\title{
Le Viêt Nam, un pays francophone atypique : regard sur l'emprise française sur l'évolution littéraire et journalistique au Viêt Nam depuis la première moitié du $\mathrm{XX}^{\mathrm{e}}$ siècle
}

\section{Thu Hang Le}

\section{OpenEdition}

\section{Journals}

Édition électronique

URL : https://journals.openedition.org/dhfles/498

DOI : $10.4000 /$ dhfles.498

ISSN : 2221-4038

Éditeur

Société Internationale pour l'Histoire du Français Langue Étrangère ou Seconde

Édition imprimée

Date de publication : 1 janvier 2008

Pagination : 341-350

ISSN : 0992-7654

Référence électronique

Thu Hang Le, "Le Viêt Nam, un pays francophone atypique : regard sur l'emprise française sur l'évolution littéraire et journalistique au Viêt Nam depuis la première moitié du XXe siècle », Documents pour l'histoire du français langue étrangère ou seconde [En ligne], 40/41 | 2008, mis en ligne le 17 décembre 2010, consulté le 27 mai 2021. URL : http://journals.openedition.org/dhfles/498 ; DOI : https://doi.org/10.4000/dhfles.498

Ce document a été généré automatiquement le 27 mai 2021

(C) SIHFLES 


\title{
Le Viêt Nam, un pays francophone atypique : regard sur l'emprise française sur l'évolution littéraire et journalistique au Viêt Nam depuis la première moitié $\mathrm{du} \mathrm{XX}^{\mathrm{e}}$ siècle
}

\author{
Thu Hang Le
}

\section{La présence de la langue française dans le quốc ngữ (le vietnamien)}

1 Le Việt Nam ne se trouve pas hors du cercle de découverte des navigateurs et missionnaires européens au XVI ${ }^{e}$ siècle. Les rivages cochinchinois sont successivement découverts par des marchands hollandais et des missionnaires dominicains, franciscains et jésuites. Un grand obstacle les attend : le langage. Le pays possède deux systèmes d'écriture différents: les caractères chinois sont destinés aux lettrés qui doivent passer presque la moitié de leur vie à les étudier; et le nôm, l'écriture dite démotique du langage quotidien, paraît plus compliqué parce qu'il associe des caractères empruntés au chinois. Il arrive très souvent que la population ne sache pas écrire sa langue. Les missionnaires inventent donc la notation destinée à écrire avec des caractères latins les sons de la langue vietnamienne, le quốc ngữ. Commencée par le jésuite natif du Portugal Francisco de Pina, régularisée et codifiée par le jésuite avignonnais Alexandre de Rhodes, puis perfectionnée par Pigneau de Behaine, cette transcription en caractères latins commence d'abord à servir aux prédications, puis est enseignée peu à peu par les Pères aux fidèles vietnamiens.

2 Lors de l'installation française en Cochinchine en 1861, la création du quốc ngũ s'achève déjà. La nouvelle administration découvre aussitôt ses avantages prédominants. D'abord, il est possible de le maîtriser en un court temps. De plus, sa ressemblance avec 
le français favorisera davantage l'accès à son apprentissage au point que l'Administration cherche à le diffuser largement. Enfin, les autorités ne cachent pas leur ambition de couper le Việt Nam de l'influence chinoise. Cette œuvre sera alors commencée par l'établissement d'une éducation à la française qui négligera peu à peu l'enseignement des caractères et lui préférera celui du quốc ngũ et du français. Les derniers concours triennaux perdent de plus en plus leur caractère traditionnel lorsque les autorités coloniales insèrent dans leur programme la connaissance de ces écritures latines, bien que facultatives. En 1919, le sort des concours triennaux est finalement jeté : le dernier concours triennal a lieu à Huế, alors qu'il a été supprimé au Tonkin dès 1915. C'est l'enseignement moderne qui se popularise désormais.

3 En effet, la création des écoles occidentales jusqu'aux villages est initiée dès 1865 par l'amiral de La Grandière. Plusieurs réformes éducatives sont successivement élaborées dans le but de trouver de meilleures solutions à la position de la langue française. Pendant trente ans (1876-1906), en Cochinchine, l'éducation publique est uniquement organisée en vue d'enseigner le français dès la première classe. Le Gouvernement met en pratique un programme qu'il ne prétendra pas nommer «assimilation» ou "francisation" des Vietnamiens, mais qui doit plutôt leur apporter l'évolution rationnelle de leur mentalité, l'amélioration de leurs qualités natives et la culture de leurs aptitudes par une éducation appropriée. Les résultats sont pourtant désastreux. Les enfants apprennent la langue qu'ils oublient aussitôt retournés à la rizière paternelle, car dans certaines provinces, il n'y a pas dix Français pour 100000 habitants.

4 À partir de 1906, la réforme de l'éducation décide que le français est facultatif dans l'enseignement primaire et que les cours en français ne pourront être donnés qu'à partir de l'enseignement primaire supérieur (équivalent du collège). À partir de cette période, la diffusion du quốc ngũ obtiendra non seulement le soutien du Gouvernement mais aussi celui des patriotes vietnamiens dans leur lutte pour l'indépendance du pays.

Dès les premières années $\mathrm{du} \mathrm{XX}^{\mathrm{e}}$ siècle, on aperçoit de nouveaux termes et notions qu'ignore le vocabulaire vietnamien. Certes cette implantation doit sans doute aux livres dits « Nouvelles Lettres » (Tân thu'), traduits du chinois, évidemment interdits par les autorités. En même temps, les marchandises de consommation françaises ne tardent pas à arriver au Việt Nam. Le vocabulaire vietnamien se montre de nouveau insuffisant pour pouvoir les nommer. Il en résulte que le quốc ngũ s'enrichit et se perfectionne, d'une part en recourant au chinois pour les termes politiques, économiques : văn minh (civilisation), công nghệ (industrie), cách mạng (révolution), dân chủ (démocratie), etc., et d'autre part en empruntant ou bien en vietnamisant le français pour l'appellation des objets nouveaux : xoong (casserole), cồn (alcool), phó mát (fromage), ray (rail), ga (gare), ô tô (auto), canô (canot), etc., et les noms scientifiques, en particulier dans la chimie : axít, a-luy-min, amin, anilin, etc.

6 À partir de 1945, la campagne d'alphabétisation s'effectue à une grande échelle à l'appel du Gouvernement provisoire du Việt Nam. À dater de 1955, le quốc ngũ s'introduira comme langue véhiculaire dans tous les degrés de l'enseignement, en remplaçant le français. Cette particularité linguistique rend exceptionnel le Việt Nam dans le monde francophone. En 1970, le Việt Nam adhère à l'Organisation internationale de la Francophonie. Le pays de 83 millions d'habitants compte cependant moins de $1 \%$ de francophones (150000 francophones réels, 200000 francophones occasionnels et 200000 apprenants le français (considérés comme francophones partiels). 


\section{L'influence française sur la presse}

7 Il est intéressant de mentionner que la presse n'existait pas au Việt Nam avant l'installation française. L'information est diffusée dans la société ancienne par des moyens relativement rudimentaires: au niveau national, le messager spécialement envoyé par le roi assume l'expédition des édits, et localement, les habitants du village sont informés soit par les affiches à la maison commune, soit par le crieur public. Il n'existait pas de moyen d'information de masse par manque de technique d'imprimerie puissante et rapide. Jusqu'à l'établissement français, l'impression s'effectue en xylographie, une technique à la fois artisanale et coûteuse.

8 Peu après l'arrivée du contre-amiral Bonard $(1861)^{1}$, la première imprimerie, l'Imprimerie impériale ${ }^{2}$, est créée à Sài Gòn, assumant toutes les publications de l'Administration. Sans doute a-t-elle renouvelé complètement les procédés élémentaires d'information pratiqués auparavant.

9 Les premiers périodiques sont aussitôt fondés dans le but d'informer sur la France et aussi les Européens en Cochinchine des politiques du Gouvernement. Quoique rédigés en français (les Bulletins, les Annuaires et le Courrier de Saigon (18633), ou bien en quốc ngũ̃, (le Gia-định báo, Sài Gòn, 1865, le Nông-cổ mín-đàm, Sài Gòn, 1900, le Đại-Nam đồngvăn nhật-báo, Hà Nôi, 1892, etc.), ils sont plutôt officiels et sont fortement inspirés des journaux officiels de la Métropole. De nombreuses feuilles privées ne tardent pas à paraître, mais elles ne diffèrent pas considérablement des titres de l'État. D'ailleurs, elles reproduisent très souvent dans les deux premières pages, contre les subventions versées par le Gouvernement, les informations, les textes officiels transmis par le Gouvernement et la Cour impériale, ou encore de longs essais sur la morale, la philosophie ou les systèmes culturels.

10 Pendant plus d'un demi-siècle (1861-1932), ces périodiques ne connaissent pas de changements considérables en matière technique. La présentation de ces journaux est relativement simple. Les articles sont successivement présentés, en fonction du format, en deux, trois ou cinq longues colonnes, sans illustrations, remplies de caractères troublant sans doute la lecture.

11 D'ailleurs, le style journalistique est loin de parvenir à la même fluidité que son homologue français. Rappelons, en effet, que le quốc ngũ̃ ne servait auparavant qu'à la diffusion de l'Évangile, qui était en outre dédaigné par les lettrés Vietnamiens, étant donné leur hostilité vis-à-vis de cette religion exotique, ainsi que la supériorité des caractères chinois. Ce début de la presse en quốc ngũ doit être plutôt considéré comme une étape d'expérimentation des capacités de ces deux éléments complètement nouveaux au Việt Nam. Sans doute le style reste-t-il très académique et archaïque, plein d'inspiration chinoise classique avec des longueurs et des parallélismes dans les phrases, des réminiscences et des allusions littéraires.

12 En général, la dernière page des périodiques est entièrement réservée à la publicité. Cette pratique était absolument ignorée et étrangère sous n'importe quelle forme au Việt Nam. Le Journal officiel ${ }^{4}$ et le Bulletin officiel reçoivent eux-mêmes les publicités. Leurs clients sont uniquement les commerçants français (les imprimeries, les concessionnaires d'automobiles, les magasins de vin ou les pharmacies, etc.) jusqu'aux années 1920, lors de la naissance de quelques commerces vietnamiens. Les publicités 
paraissent de plus en plus sophistiquées. Si elles consistent au début en simples écrits, les illustrations, d'abord en noir et blanc, puis en couleur, y seront insérées. Pourtant tous les journaux en quốc ngũ ne connaissent pas ce raffinement technique avant 1927.

En effet, l'an 1927 marque un grand changement dans la presse vietnamienne grâce à deux ambitieux de "retour de France ", Hoàng Tích Chu, le rénovateur du style journalistique, et Đỗ Văn, de la technique d'imprimerie, avec qui les fonctions de la presse et du métier de journaliste seront clairement définies. Leur esprit d'initiative et d'originalité audacieuse, les connaissances acquises lors de leur séjour d'apprentissage en France offrent au quotidien Hà thành ngọ báo (le $1^{\mathrm{er}}$ juin 1927) devenant Ngọ báo (Le midi) de Bùi Xuân Học, plus tard à leur propre journal hebdomadaire Đông Tây, une présentation qui fait penser aux plus grands quotidiens de France.

À partir de cette date historique, la presse vietnamienne se développe contrairement à la routine persistante des périodiques contemporains. Elle est, en outre, plus variée, avec l'essor de différents types de journaux spécialisés (littéraires ou culturels, scientifiques, commerciaux, d'éducation, féminins ou enfantins, etc.). Les articles sont brefs et concis. Les actualités, l'essentiel d'un quotidien, font la une. Ce nouveau style, fortement inspiré du journalisme français, n'est pas immédiatement accepté par le lecteur, voire par différents collègues de ces deux innovateurs. De nombreuses polémiques se déclarent du nord au sud du Việt Nam et divisent le monde littéraire en deux groupes: les jeunes littérateurs sont les partisans de ce nouveau souffle et les lettrés s'y opposent.

Cette bonne volonté sera renforcée en 1932 par l'enthousiasme des journaux Phong Hóa (1932-1935), puis Ngày Nay (1935-1937) du groupe " Tự Lực văn đoàn ». Il s'agit d'un tournant tellement important qu'on le dénommera la génération de 1932. En effet, de nombreux changements primordiaux, et aussi des fantaisies naissent au cours de cette époque, en particulier grâce au groupe littéraire Tự Lực.

Le courant satirique s'introduit pour la première fois dans la presse en quốc ngữ ${ }^{5}$ La nouvelle version du Phong Hóa consiste à «discuter avec humour les questions essentielles relatives à la société, la politique, l'économie, et à retracer exactement les réalités du pays $»^{6}$. Certes ce courant, évidemment bien connu en France, constitue au Việt Nam un nouveau début qui fera connaître aussitôt certains titres célèbres, Vịt Đực (Le Canard, juin 1938-juil. 1939), Con Ong (L'Abeille, mai 1939-janv. 1940), etc. Il est évident que ces journaux satiriques, qui ne flattent pas les autorités et leurs adorateurs, connaissent une existence éphémère, car le Gouverneur général a toujours le pouvoir de leur retirer l'autorisation préalable.

La presse vietnamienne à partir de 1945 continuera cette amélioration de la technique d'impression. En regardant le journal Tiếng dội (l'Écho, 1949-1954) paraissant à Sài Gòn et son confrère hanoïen Tia sáng (le Rayon lumineux, 1950-1954), il est absolument possible de les comparer aux titres contemporains paraissant en Métropole, L'Humanité ou Le Monde. La surcharge de style auparavant copiée de la littérature chinoise est complètement abandonnée. La presse joue désormais le rôle d'un organe d'information dont le ton parvient à la même fluidité que les journaux actuels. 


\section{L'influence française sur la littérature vietnamienne}

18 Une nette inspiration française est également constatée dans la littérature vietnamienne. Cela ne signifie pas que la littérature vietnamienne n'existait pas auparavant, comme la presse, mais elle ne comprenait que la prosodie, alors que la prose était apparue récemment, vers le XVIII ${ }^{\mathrm{e}}$ siècle, et était plutôt destinée à la littérature populaire. Cette littérature savante, considérée comme un signe de haute culture et de qualité du poème devait respecter les règles de la prosodie des Tang (Đường): le huitain heptamètre (sonnets de 8 vers de 7 pieds chacun) avec de nombreuses règles strictement déterminées par les parallélismes, les préciosités et l'interdiction des noms royaux.

19 Ce nouvel apport français s'exprime, en premier lieu, dans la signification du métier d'écrivain, auparavant inconnue au Việt Nam. Il s'agissait plutôt d'un passe-temps favori et subtil des lettrés qui accordaient souvent à leurs œuvres un objectif éducatif. Jusqu'aux années 1930, le métier trouve peu à peu sa place, mais il est impossible de vivre des productions littéraires et la plupart des écrivains exercent un autre métier.

Pour pouvoir parvenir à cette reconnaissance, les premières années du $\mathrm{XX}^{\mathrm{e}}$ siècle servent aux préparatifs pour la floraison littéraire née la période suivante. La diffusion de la littérature et des auteurs français est à la fois étendue et sélective. En effet, elle s'adresse d'une part au grand public grâce aux travaux inlassables des pionniers, en particulier Nguyễn Văn Vĩnh qui a traduit en quốc ngũ et publié les grands auteurs français dans le Đông Dương tạp chí (La Revue Indochinoise), puis dans la collection « Âu Tây tư tưởng " (Les Pensées Occidentales), et Phạm Quỳnh qui a élaboré, dans sa revue encyclopédique Nam Phong (Le Vent du Sud), de nombreux essais et explications sur la littérature française. Elle sera enseignée, d'autre part, aux étudiants en lettres de l'école normale supérieure qui deviendront, pour la plupart, des littérateurs célèbres.

21 En second lieu, à partir de la grande année $1932^{7}$, la littérature vietnamienne se diversifie avec l'introduction de différents courants et genres littéraires. Elle se manifeste désormais comme réformée et absolument différente de la littérature ancienne ; d'où les appellations de « Roman Nouveau » et de " Poésie Nouvelle ».

La Poésie Nouvelle s'inspire incontestablement de la versification française, de la forme à la substance : la division en strophes (khổ), comprenant ainsi les quatrains (khổ bốn câu) et tercets (khổ ba câu) ou encore, l'adoption des rimes suivies (vần liền), rimes croisées (vần chéo), rimes embrassées (vần ôm), et l'utilisation flexible des mots, des images, et la manière d'expression de la pensée.

Le romantisme et le réalisme imprègnent pour la première fois la littérature vietnamienne. Le romantisme dominera plutôt la littérature des années 1932 et 1937, correspondant à l'existence du groupe littéraire "Tự lực » qui cherche à apaiser les conséquences dues aux grandes répressions contre les révoltes éclatées dans tout le pays durant les années 1930 et 1931. Il est possible de reconnaître vaguement chez les auteurs vietnamiens Xuân Diệu, Thế Lữ, Hàn Mạc Tử, Lưu Trọng Lư, Huy Thông, Chế Lan Viên, Huy Cân, Tế Hanh, l'influence des grands poètes français, de Lamartine, Victor Hugo et surtout de Baudelaire, Rimbaud, Verlaine.

24 Le réalisme se fait connaître déjà durant cette période, à travers les genres du reportage et du roman. Les auteurs n'hésitent pas à raconter la vie des tireurs de 
pousse et des filles de joie, ce qui leur permet de reconstituer les conditions de vie et de retracer avec précision les faces oubliées de différentes couches sociales 8 .

Il semble que le réalisme l'emporte sur le romantisme à partir de 1939, au moment où le Việt Nam commence à vivre sous les deux étreintes française et japonaise. La littérature réaliste des années 1940 à 1954 continue à dénoncer le joug des notables mis en place par les mandarins et les colonisateurs. Là on reconnaît l'influence des auteurs véristes français, de Victor Hugo ou Hector Malot, de Henri Barbusseou André Gide, dans le récit de la misère et de la vie sans issue, pitoyable et aléatoire des exclus de la société, mais aussi des intellectuels petits-bourgeois. Durant la résistance pour l'indépendance du pays, l'écrivain et le poète deviennent des combattants, la littérature reflétant la grandeur de la cause révolutionnaire, l'héroïsme, la simplicité, la beauté humaine des militants, des ouvriers, des paysans dans toute leur richesse.

En dernier lieu, il convient de mentionner l'influence française sur l'évolution du langage littéraire. Une grande maturité se révèle manifestement entre le roman rénovateur portant la naissance du « Roman Nouveau », le Tố Tâm (Hoàng Ngọc Phách, 1925) et les œuvres d'à partir de 1932. Le triste style maniéré et la fleur bleue des romans chinois de l'époque, présents pourtant dans ce premier roman romantique, seront emportés par la nature du langage des personnages plus direct, plus concret et plus proche du parler quotidien.

\section{Conclusion}

Pendant un demi-siècle, la littérature vietnamienne se diversifie et développe tous les genres : le reportage, les essais et chroniques, les mélanges, le théâtre, les poésies et la prose se diversifiant en contes et nouvelles en prose, romans et romans de cape et d'épée, romans historiques, romans policiers.

Elle prouve la capacité de faire de la littérature en quốc ngữ, ce qui était mis en doute auparavant. D'ailleurs ces deux domaines se complètent et s'enrichissent mutuellement. L'influence française est évidente dans cette œuvre. Pourtant il est regrettable que les anciens caractères soient tout à fait abandonnés. Dès les années 1940, on s'inquiétait déjà de l'ignorance et de l'occidentalisation des jeunes. Cette lacune devient plus importante de nos jours, car à part une petite partie d'apprenants des han et nôm, personne ne peut décoder les livres anciens uniquement rédigés en idéogrammes.

\section{BIBLIOGRAPHIE}

Annuaire de la Cochinchine française pour l'année 1866, Saigon, Imprimerie impériale, 1866.

BAUDRIT, André (éd.) (1936). Contribution à l'histoire de Saigon, extraits des registres de délibérations de la ville deSaigon (Indochine française) 1867-1919, Saigon, Imprimerie J. Testelin. 
BROCHEUX, Pierre \& HÉMERY, Daniel (2001). Indochine : la colonisation ambiguë 1858-1954, Éditions La Découverte, 2001.

LAFONT, Pierre-Bernard (1992). Les emprunts au vocabulaire français dans la langue vietnamienne, The Edwin Mellen Press.

Lê Thước (1921). L'enseignement des caractères chinois, Hà Nội-Hải Phòng, Imprimerie d'ExtrêmeOrient.

Phạm Thế Ngũ, Việt Nam văn học sử giản ước tân biên, rééd., California : Đại Nam, avant 1975.

Vũ Ngọc Phan (1989). Nhà văn hiện đại, rééd., Thành phố Hồ Chí Minh : Khoa học xã hội.

\section{NOTES}

1. Avant de quitter la France, Bonard a prévu d'établir en Cochinchine une imprimerie et a chargé le capitaine de frégate Lavaissière de Lavergne, qui devait le rejoindre un mois après, d'apporter un appareil avec quelques ouvriers.

2. L'Imprimerie impériale est fermée en 1868. À partir de 1869, les publications officielles seront en charge de l'Imprimerie du Gouvernement.

3. Le gérant du journal est $\mathrm{C}$. $\mathrm{C}$. Tissier. La Bibliothèque nationale de France le conserve à partir du $10^{\mathrm{e}}$ numéro. Ce numéro mentionne que le Courrier de Saigon paraît le 10 et 25 de chaque mois. Le premier numéro doit donc paraître le 25 décembre 1863.

4. On constate la publicité de l'imprimerie L. Gallois durant l'an 1907 dans le Journal officiel de l'Indochine française.

5. Ce courant a été déjà employé par quelques propriétaires français dès la fin du XIX ${ }^{\mathrm{e}}$ siècle dans leur but personnel, très souvent pour critiquer leur adversaire.

6. Phong Hóa, $\mathrm{n}^{\circ} 13$, jeudi 8 septembre 1932 : publicité pour la nouvelle version du Phong Hóa à partir du $14^{\mathrm{e}}$ numéro du 22 septembre 1932.

7. Nommée également la génération de 1932, elle marque un tournant important de l'évolution intellectuelle vietnamienne. Plusieurs raisons peuvent l'expliquer: l'intronisation du jeune Empereur Bảo Đại diplômé de France, la démoralisation de la population due à la répression des révoltes paysannes des années 1930 et 1931, le retour de France de nombreux étudiants vietnamiens, la sortie des premiers licenciés ès lettres, en particulier la diffusion de plus en plus large, quoique légale ou clandestine, des connaissances nouvelles, etc.

8. On parlera encore pendant longtemps des reportages de Tam Lang, alias de Vũ Đình Chi, Tôi kéo xe (Je tire les pousses, 1932) et Đêm sông Hương (Nuits sur la Rivière des Parfums), de Vũ Trọng Phụng, Cạm bẫy người (Piège pour hommes) et Kỹ nghệ lấy Tây (L'industrie du mariage avec les Français, 1934).

\section{RÉSUMÉS}

La présence française au Việt Nam remonte à 1862 lors de l'installation définitive de la France en Cochinchine. Parmi les éléments introduits par la présence française, l'imprimerie, les imprimés et la littérature française entraînent, notamment grâce à la généralisation du quốc ngữ, de nombreux changements socioculturels au Việt Nam. Loin d'être une simple imposition, les 
Vietnamiens se réapproprient ces principes et les utilisent sur le plan technique mais aussi sur le plan littéraire, parfois à l'encontre des colonisateurs. Très rapidement des récits en français sont publiés qui, d'abord dans un style heurté et maladroit, vont ensuite donner naissance dans les années 1930 et 1940 à une littérature très riche, que l'on peut considérer comme un genre à part entière. Cette période est une des plus prolifiques de la culture vietnamienne et ses écrits restent encore une référence littéraire, jusqu'à aujourd'hui.

The start of French colonization in Việt Nam can be dated to 1862 when the French officially took possession of Cochin China. Among the many innovations brought by the French, industrial printing, mass publication, and French literature, and the accompanying dissemination of quốc ngũ in particular, brought about numerous social and cultural transformations. Far from being a one-way imposition, many Vietnamese appropriated the new printing principles, sometimes to anti-colonial ends, both for their technical properties and literary qualities. Shortly after the inception of colonization, Vietnamese writers began to publish narratives in French. Though this movement was characterized at first by a clumsy, uneven style, it later gave birth in the 1930s and 1940s to an incredibly rich literature that formed a coherent genre. This period was one of the most prolific in Vietnamese literature and its writings are to this day regarded as a literary standard in Việt Nam

INDEX

Mots-clés : Viêt Nam, présence française, Cochinchine, littérature, culture, vietnamienne

Keywords : Viêt Nam, French presence, Cochin China, literature, Vietnamese culture

\section{AUTEUR}

\section{THU HANG LE}

Doctorante, Université de Versailles Saint-Quentin-en-Yvelines

Centre d'histoire culturelle des sociétés contemporaines

France

le.thuhang@yahoo.fr 